\title{
THE MECHANISM OF INFLUENCE OF NON- OCTAHEDRAL SLIP ON ROLLING TEXTURE DEVELOPMENT IN FCC METALS. COMPUTER SIMULATION
}

\author{
S. V. DIVINSKII and V. N. DNIEPRENKO \\ Institute of Metal Physics, Ukrainian Academy of Science 36 Vernadsky str., \\ Kiev-142, 252680, Ukraine
}

(30 September, 1992)

\begin{abstract}
Simulation of the copper-type rolling texture development in FCC metals based on homogeneous slip under conditions of no constrains (Sachs-type model) is presented. Detailed analysis shows that, in fact, effective activation of a few (two or three, sometimes greater) independent slip systems occurs after reaching of some strain. These slip systems act by turns and may be essentially considered as acting simultaneously. Therefore, such extended description may be considered as a model which is intermediate between Taylor and Sachs ones. Taking these results into account, the characteristic features of main texture component development in copper under rolling have been studied by a computer simulation. Both octahedral, $\{111\}$, and cubic, $\{100\}$, slip planes are shown to act simultaneously in the process of the $\{112\}\langle 11 \overline{1}\rangle$ component formation, but the action of only $\{111\}\langle 1 \overline{1} 0\rangle$ slip systems is characteristic for the $\{110\}\langle 1 \overline{1} 2\rangle$ component formation. The important role of the non-octahedral sip systems in plastic deformation processes in FCC metals of high staking-fault energy are also confirmed by the coincidence of model shear textures and experimental surface textures.
\end{abstract}

KEY WORDS Computer modelling, plastic deformation, octahedral slip, cubic slip, FCC-metals.

\section{INTRODUCTION}

Computer simulation of texture formation under plastic deformation in FCC metals was the aim of numerous investigations. The results of some of them were reviewed by Hirsch et al. (1988). Complexity of the plastic deformation processes occurring really in materials and impossibility to allow for all of them at the present time result in not very convincing agreement between the model pole figures and experimental ones.

Addition of the well known Sachs model by some new presentations acquired from experimental data is considered in the present work. It has been determined by electron microscopy in a number of papers, e.g. in Vandermeer et al. (1964), Trefilov et al. (1975), and Larikov et al. (1979), that the correlation of structure and texture development is observed during plastic deformation. Virtually each texture component can be characterized by its own type of the dislocation structure. So, Dnieprenko et al. (1982) found that two main types of dislocation structures, which are clearly distinguished in the plane normal to the rolling direction (RD) and can be attributed to the $\{110\}\langle 1 \overline{1} 2\rangle$ and $\{112\}\langle 11 \overline{1}\rangle$ preferred orientations, respectively, may be unambiguously separated in rolled 
copper. If we adopt the hypothesis of Wassermann et al. (1962) about the partial axiality of rolling textures we may restrict our analysis to consideration, mainly, of these two orientations. Orientations of the dislocation subboundary traces with the use of some ideas of Takeuchi (1970), as we consider, can determinate the slip systems which have been activated in these components. Dnieprenko et al. (1982) established that the $\{110\}\langle 1 \overline{1} 2\rangle$ texture component (called the "brass" orientation), as it might be expected, was developed through rotations induced by dislocation slips in the $\{111\}$-type planes. In turn, the development of the second main texture component, namely, $\{112\}\langle 11 \overline{1}\rangle$ or copper-type orientation, can be explained with the allowance for extra slips in the $\{100\}$ planes. The direct electron-microscopical evidence of the existence of such slip type was presented, e.g., by Karthaller (1972). More earlier, the assumption of possible copper-type component development as a result of the only $\{100\}$-type slips was suggested by Haessner (1963) with hypothetical consideration of the crystallographic aspects of slips. Nevertheless, in the Taylor-type computer simulation of the texture development carried out by Wierzbanowski et al. (1984) the allowance for $\{100\}$ slips did not result in any substantial changes of the final crystal distribution in comparison with the use of only $\{111\}$ slips.

Researches give preference usually to the Taylor model, since the Sachs model leads to plastic strain incompatibility at the boundaries of adjacent grains, see Hirsch et al. (1988). Moreover, it is considered that deformation of polycrystals by single slip events as in the Sachs model, does not take place. Though, there are experimental data which indicate that only two or three slip systems really act inside grains and their total number is greater only in an immediate vicinity of the grain boundaries, Dillamore et al. (1964). This disagrees with both models. However, we may note that the resulting action of a few slip systems may be obtained within the Sachs approach e.g. due to the activation of new slip systems under increasing strain. If this is correct the Sachs model may probably not contradict the above experimental data particularly as concerning a total number of the active slip systems, whereas it is difficult to relate the Taylor model with this experiment. In the recently developed model of Zolotarevski et al. (1989) the rotations arising due to the strain incompatibility on the boundaries of adjacent grains were taken into account. In some approximation, this can be considered as the compromise between the Taylor and Sachs approaches. Main feature of the approach of Zolotarevski et al. (1989) is to allow for modern disclinational presentations, e.g. see Romanov et al. (1983), about the mechanisms of grain re-orientations.

In the present work the calculations were carried out based on the Sachs technique and also based on the model of Zolotarevski et al. (1989) in view of the above-mentioned remarks about the Taylor model and the results of Wierzbanovski et al. (1984). Nevertheless, the results of simulation by the technique of Zolotarevski et al. (1989) were obtained to be similar to those of the Sachs-type simulation. This is probably due to the fact that Zolotarevski et al. (1989) paid attention mainly to mechanisms of grain re-orientations and in a smaller extent to the slip systems activations. In the present paper the Sachs model is used to study the activation of the slip systems of different types for different initial grain orientations.

For convenience a family of slip systems with crystallographically equivalent slip planes will be further called the slip system of specific type. 


\section{MODEL}

12 octahedral slip systems $\{111\}\langle 1 \overline{1} 0\rangle$ and 6 cubic slip systems $\{100\}\langle 011\rangle$ were the slip systems considered in the computer model. Only one slip system with maximum loading was selected as the active one in each elementary act of the simulation of plastic deformation. The influence of the Schmid orientation factor $\Phi^{(s)}$ for the $s$ th slip system

$$
\Phi^{(s)}=\sum_{i, j=1}^{3} n_{i}^{(s)} \cdot \sigma_{i j} \cdot b_{j}^{(s)}
$$

was taken into account with this aim. Here $\bar{n}^{(s)}$ is the unit vector normal to the slip plane, and $\bar{b}^{(s)}$ is the unit vector coinciding with the slip direction; $\sigma$ is the stress tensor.

More precisely, the slip system with maximum value of $\Phi^{(s)} \cdot v^{(t)}$, where $v^{(t)}$ is the efficiency of activation of the $t$ th type slip system (i.e. $v^{\text {(cub) }}$ for cubic $\{100\}\langle 011\rangle$ systems and $v^{(\mathrm{oct})}$ for octahedral $\{111\}\langle 1 \overline{10}\rangle$ ones), is considered as the active one in this work. In the first approximation the choice of the $v^{\text {(cub) }}$ and $v^{(\mathrm{oct})}$ values may be based on the results of dislocation energy calculations for different crystallographic planes carried out by Foreman (1955). In that work the elastic energies of dislocations in the (100) and (111) planes of FCC metals (for example copper) were shown to differ by the $10 \%$ owing to the lattice elastic anisotropy, energy of the dislocation core and the dislocation splitting may introduce some corrections in this value. In frames of our model the $v^{\text {(cub) }} / v^{\text {(oct) }}$ ratio effects, mainly, the volume ratio of texture components and, therefore, may be determined by the correlation of component compositions of the model and experimental textures.

After the slip system had been selected, a certain amount of slip was performed (in the present calculations the amount of shear per slip event was varied in the range $0.01-0.05$ ). The rotation accompanying each slip event was computed as by Leffers (1968a). The rotation, as performed, fulfills two requirements of Leffers (1968b): (a) a string of material lying in the rolling direction before slip must retain this orientation when the slip has taken place, and (b) a plate of material lying in the rolling plane before slip must retain this orientation. The calculations in a stepwise manner (slip system choice-rotation-slip system choicerotation ...) has been carried out until the desired reduction of the thickness of the crystal was obtained. In a majority of the calculations the number of the crystals in question was 300 .

\section{THE ROLLING TEXTURES OF THE INNER LAYERS}

The results presented below were obtained with using of the routine biaxial stress tensor, where only $\sigma_{x x}=-\sigma_{z z} \neq 0$ (here $O x$ coincides with the $\mathrm{RD}$ and $O z$ coincides with the normal to the rolling plane). The simulation showed that small additions of various shear components cause no significant changes of the results obtained.

Let us suppose that the deformation proceeds only by crystallographic slip in slip systems of the $\{111\}\langle 1 \overline{1} 0\rangle$ type. In this case the model texture is described mainly by the $\{110\}\langle 112\rangle$ component, see Figure 1a. The weak $\{110\}\langle 001\rangle$ component may also be detected particularly on the $\{100\}$ pole figure (Figure 1a). 

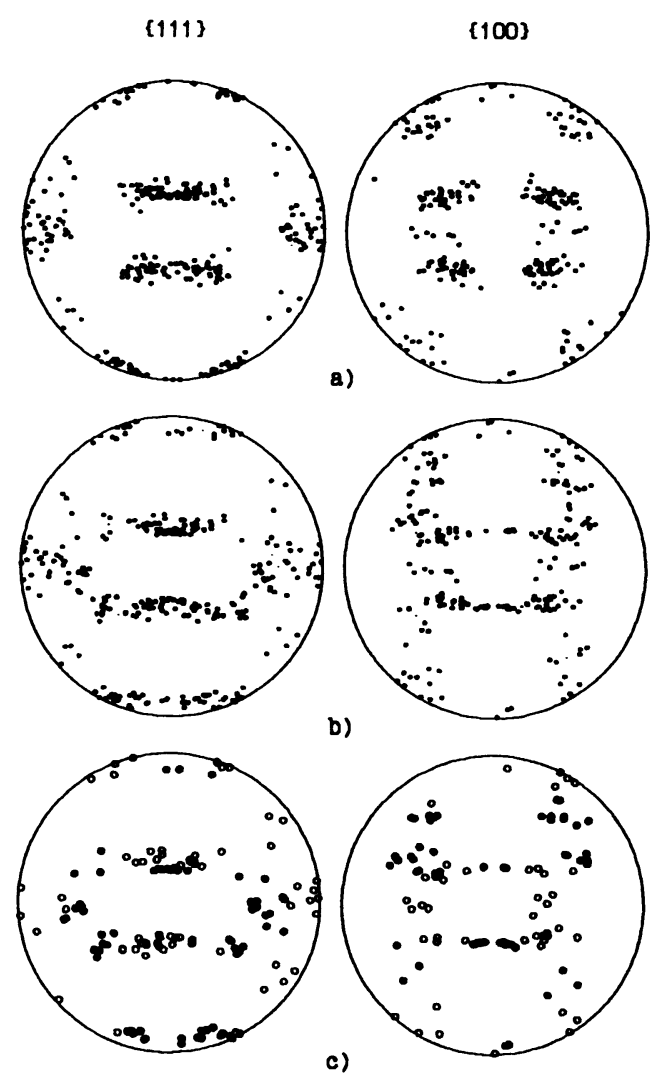

Figure 1 Calculated pole figures for the $\{111\}\langle 110\rangle$ active systems (a) and for $\{111\}\langle 110\rangle$ plus $\{100\}\langle 011\rangle$ active systems (b), the same with allowance for specific deformation mechanisms (c). $60 \%$ reduction. In (c) the grain orientations are marked by special symbols in dependence on the slip systems acting in the last deformation stages, namely $\{111\}\langle 1 \underline{1} 0\rangle+\{100\}\langle 011\rangle(\cdot) ; \quad\{111\}\langle 1 \overline{1} 0\rangle+$ $\{111\}\langle 1 \overline{10}\rangle\left({ }^{\circ}\right)$.

Addition of the $\{100\}\langle 011\rangle$ slip systems as a possible mechanism of plastic deformation results in the development of an extra component in addition to the ones mentioned above which is close to $\{112\}\langle 111\rangle$, and, therefore, the copper-type texture is formed, see Figure $1 \mathrm{~b}$. Coincidence of the model component composition with the experimental one for rolled copper is reached at $v^{(\mathrm{cub})} / v^{(\mathrm{oct})}=0.87$. Note, that the reverse relationship, namely, $v^{\text {(cub) }} / v^{(\mathrm{oct})}>1$, had to be assumed accounting for only elastic energy of perfect (non-split) dislocations by Foreman (1955). Probably the factors, which were not considered by Foreman (1955), particularly dislocation splitting, diminish the dislocation energy in the octahedral planes in comparison with that for the cubic planes. This results in a higher efficiency of activation of slip systems of the $\{111\}\langle 110\rangle$ type comparing with those of the $\{100\}\langle 011\rangle$ type.

If we take into account slip systems only of the cubic type a texture reminding the one presented in Figure $1 \mathrm{a}$ and typical for $\{111\}\langle 110\rangle$-type slip will be developed. Thus, the copper component in Figure $1 \mathrm{~b}$ is developed by the way which differs from simple slips in the cubic planes as it might be expected. For establishing the nature of the development of this component a more detailed analysis is to be carried out. 

COMPONENT DEVELOPMENT

The particular slip types causing the development of the above mentioned components of the copper rolling texture are studied in this part of the work. For this aim we will monitor the indices of the slip system with maximum loading (which will be active at this moment) during every elementary act of the plastic deformation and for every grain. Plastic deformation by sequential slips in a single slip system was found to be replaced by alternative slips in a few slip systems after reaching the average strain of $\sim 30 \%$. It should be remembered that the Sachs model, for which a single slip system is active in each elementary act, is used.

It was obtained that the paths of development of the $\{110\}\langle 1 \overline{1} 2\rangle$ and $\{112\}\langle 11 \overline{1}\rangle$ main texture components are principally different. The $\{110\}\langle 1 \overline{1} 2\rangle$ component is developed as a result of the alternative action of at least two octahedral slip systems, e.g. (111)[110] and (111) [101], whereas the copper component is developed by alternative slips in the $\{111\}\langle\overline{1} 10\rangle$ and $\{100\}\langle 011\rangle$ slip system types. In Figure 2 the model orientation distribution function (ODF) of grains as a function of the Eulerian angles $\left(\varphi_{1}, \Phi, \varphi_{2}\right)$ is presented, the grain orientations being marked by special symbols which characterize the sequence of slips acting during medium and final stages of the deformation. The regularity of the slip system changes was studied for this marking. The last 25 elementary deformation steps (before reaching the given reduction) were recorded. Let $c$ and o denote some slip systems of the cubic and octahedral type, respectively. If $\mathrm{c} \rightarrow \mathrm{c} \rightarrow \mathrm{c} \rightarrow \mathrm{c} \ldots$ (or $\mathrm{o} \rightarrow \mathrm{o} \rightarrow \mathrm{o} \rightarrow \mathrm{o} \ldots$ ) slip system change is detected on the last deformation stages, then this corresponds to the action of a single slip system of

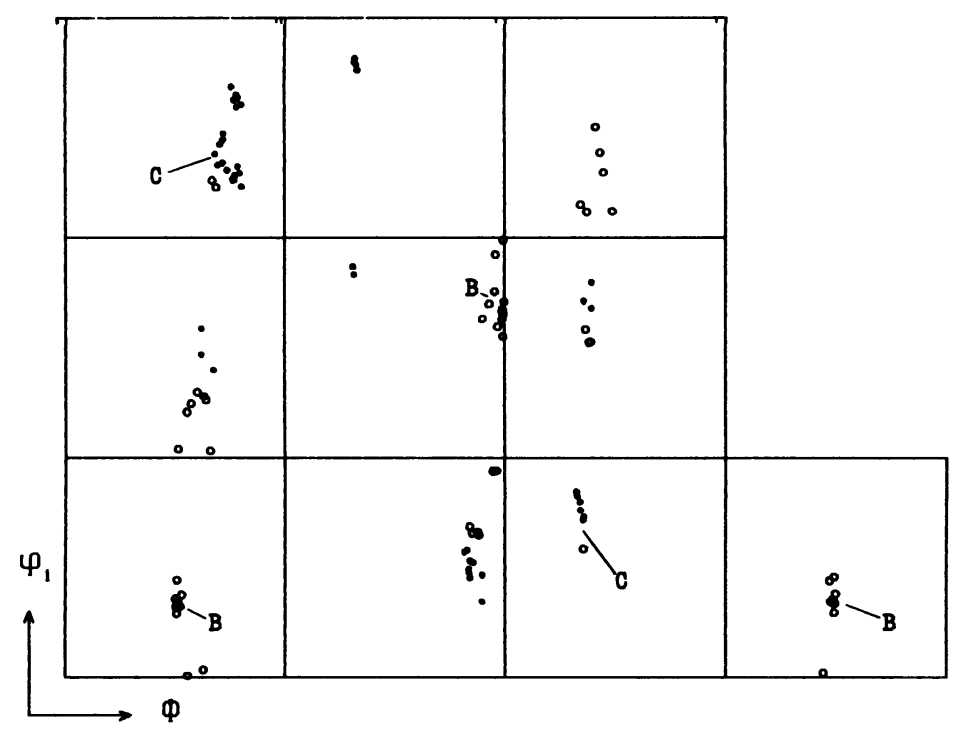

Figure 2 Calculated ODF for $\{111\}\langle 1 \overline{1} 0\rangle$ plus $\{100\}\langle 011\rangle$ active systems. The components indicated are the copper-type $(C)$ and brass-type $(B)$ ones. The grain orientations are marked as in Figure 1c. 


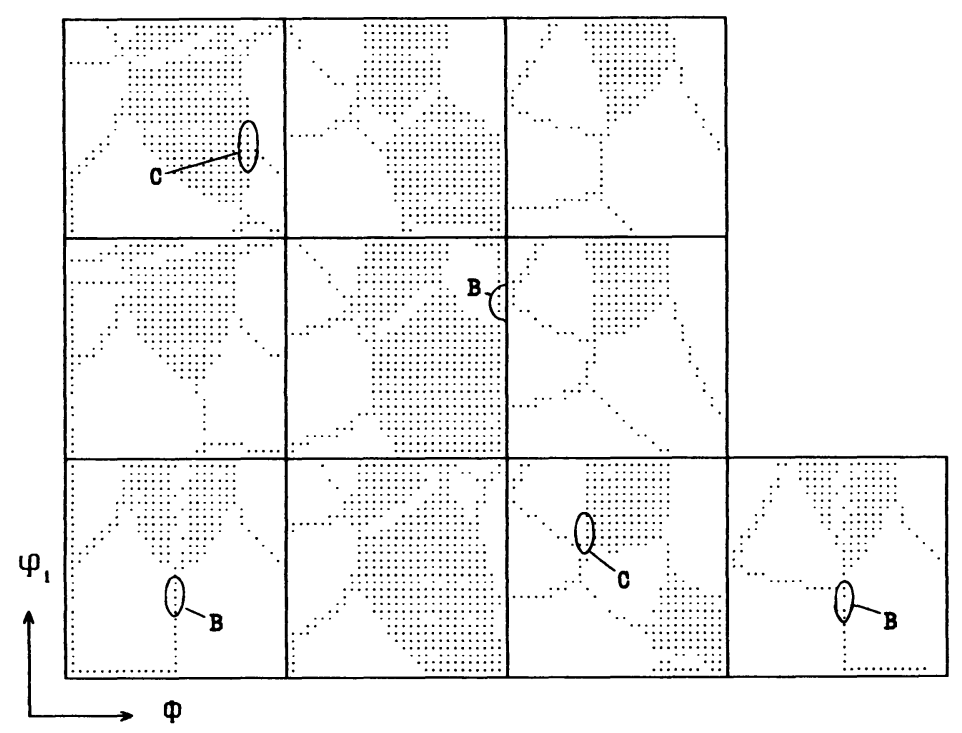

Figure 3 The regions of action of different slip systems: the $\{111\}\langle 1 \overline{1} 0\rangle$ type (bright) and the $\{100\}\langle 011\rangle$ type (dark). The $\mathrm{B}$ and $\mathrm{C}$ symbols indicate the corresponding regions of stability of the grain orientations to rolling in dependence on the predominant orientations (see Figure 2).

the $\{100\}\langle 011\rangle$ (or $\{111\}\langle 1 \overline{1} 0\rangle)$ type. The $c \rightarrow c^{\prime} \rightarrow c \rightarrow c^{\prime} \rightarrow c \ldots$ (or $o \rightarrow o^{\prime} \rightarrow$ $o \rightarrow 0^{\prime} \rightarrow \mathbf{o} \ldots$. ) chain means the alternative action of two different slip systems of the same type and is marked as $\{100\}\langle 011\rangle+\{100\}\langle 011\rangle$ (or $\{111\}\langle 110\rangle+$ $\{111\}\langle 1 \overline{1} 0\rangle)$. The $\mathrm{c} \rightarrow \mathrm{o} \rightarrow \mathrm{c} \rightarrow \mathrm{o} \rightarrow \mathrm{c} \ldots$ chain signifies the alternative action of slip systems of different types and is marked as $\{111\}\langle 1 \overline{1} 0\rangle+\{100\}\langle 011\rangle$ (the $\mathrm{c} \rightarrow \mathrm{o} \rightarrow \mathrm{o}^{\prime} \rightarrow \mathrm{c} \rightarrow \mathrm{o} \rightarrow \mathrm{o}^{\prime} \rightarrow \mathrm{c} \ldots$ cases are also included here). The $\{111\}$ and $\{100\}$ pole figures, which were presented by standard means without any separation of grains according to the deformation mechanisms, are shown in Figure 1c using the same symbols as in Figure 2 to demonstrate clearly the advantages of our approach.

The detailed analysis of the number of the quasi-simultaneously active slip systems revealed that both two and three independent slip systems may act together during the last deformation stages. This is now in agreement with the experimental data of Dillamore et al. (1964). For example, the following combination was observed: (111) [011], (1111) [110], and (001)[110].

The main components of the copper-type rolling texture would develop near the boundaries of regions of action of the different slip systems as the analysis carried out shows. These regions are pointed in Figure 3, the system with the maximum value of $\Phi^{(s)} \cdot v^{(t)}$ being considered as the active one. Systems with $\{111\}$ and $\{100\}$ type slip planes were taken into account. The positions of the preferred orientations (Figure 2) are in agreement with the regions of grain orientation stability to such slip. These regions are also marked in Figure 3 . The divergence of a grain orientation from the initial location after a given number of deformation steps were studied for analyzing this stability. The angular distance $\omega$ between an initial grain orientation $\varphi_{1}^{0}, \Phi^{0}, \varphi_{2}^{0}$ and the current one $\varphi_{1}, \Phi, \varphi_{2}$ 
was computed as

$$
\begin{aligned}
\cos \omega= & 0.5\left[\cos \left(\varphi_{1}-\varphi_{1}^{0}\right) \cos \left(\varphi_{2}-\varphi_{2}^{0}\right)\left(1+\cos \Phi \cos \Phi^{0}\right)\right. \\
& -\sin \left(\varphi_{1}-\varphi_{1}^{0}\right) \sin \left(\varphi_{2}-\varphi_{2}^{0}\right)\left[\cos \Phi+\cos \Phi^{0}\right] \\
& \left.-\left[\cos \left(\varphi_{1}-\varphi_{1}^{0}\right)+\cos \left(\varphi_{2}-\varphi_{2}^{0}\right)\right] \sin \Phi \sin \Phi^{0}+\cos \Phi \cos \Phi^{0}-1\right]
\end{aligned}
$$

The $\omega$ value after reaching $10 \%$ reduction was used as the measure of the stability, and it was computed step-by-step for every grain orientation with $5^{\circ}$ steps for all three Eulerian angles. In this case the shear per slip event was 0.01. The regions with $\omega<1^{\circ}$ are marked in Figure 3 as the stable ones.

It can be noted that the spread of the grain orientations in the direction perpendicular to the boundary of the regions of the action of different slip systems is clearly smaller than the spread along this boundary, see Figures 2 and 3. This is in agreement with Wassermann's assumptions mentioned above about a partial axiality of the textures. Moreover, the copper component is clearly seen to have the greater "axiality" (the relation of the spreads in the two mutually perpendicular directions mentioned above) than the brass component.

Thus, textures may be quite strictly resolved into separate components even with allowance for their spreading up to overlapping. The specific deformation mechanism of each grain plays the key role for this.

\section{THE SURFACE LAYERS' TEXTURES}

Existence of slip systems of the $\{100\}\langle 011\rangle$ type allows to explain the abnormal surface textures of FCC metals of high stacking fault energy (SFE).

For rolled $\mathrm{Cu}$ the texture of the surface layers, where in general a shear stress components acts, is known to be described mainly by the $\{100\}\langle 011\rangle$ preferred orientation, see Regenet et al. (1963) and other. The crystallographic indices of this orientation are explicitly seen to coincide with the ones of the slip plane and of the Burgers vector of dislocations for the cubic-type slip systems. Therefore, one may suppose that it is the very type of slip that causes the development of the above mentioned surface texture.

Within the present hypothesis the simulation of the texture arising in the surface layers was carried out. The only shear component connected with a shear in the plane involving both the rolling and normal direction differs from zero in the model stress tensor.

In Figure 4 the shear stress simulation results are presented.

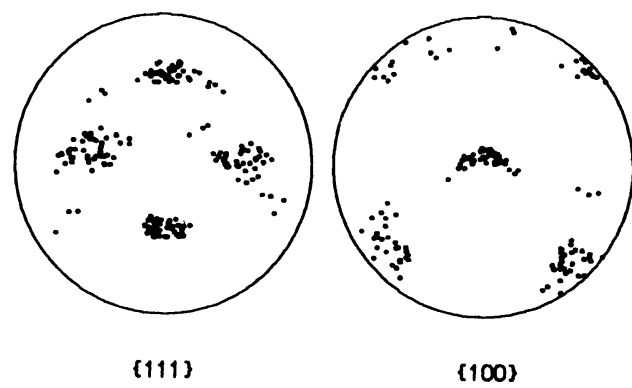

Figure 4 Calculated pole figures for coppertype surface layer texture. $\varepsilon=60 \%$. 
A similar texture $\{100\}\langle 011\rangle$ was simulated by only $\{111\}\langle 1 \overline{1} 0\rangle$ slip by Wierzbanovski et al. (1982) accounting for a change of shear components of the deformation tensor along the roll gap, determined experimentally by other researchers. The extreme case corresponding to large reduction per deformation step was considered by Wierzbanovski et al. (1982). Our simulation in frames of his model shows that the calculations based on the experimental data for standard reductions per step do not result in the $\{100\}\langle 011\rangle$ texture development without allowances of any possibility of slips in the cubic planes.

\section{CONCLUSIONS}

Thus, the results of the computer simulation of the surface and inner layers textures prove the fundamental role of non-octahedral slip in the development of the rolling texture components of FCC metals of high SFE.

To summarize we may conclude:

- the $\{112\}\langle 11 \overline{1}\rangle$ component in the copper-type texture is developed as the result of the alternative action of cubic and octahedral slip systems, whereas the $\{110\}\langle 112\rangle$ component is developed by the alternative action of two or three independent octahedral slip systems;

-allowing for cubic slip and monitoring the active slip system in each elementary act of plastic deformation leads to a new insight in understanding the nature of development of the different texture components;

- the results of the present work allow to relate the following different aspects experimentally observed: (i) existence of two types of dislocation structures; (ii) activation of nonoctahedral slip; (iii) action of two or three independent slip systems; (iiii) Wassermann's assumptions on describing the textures with partial axial components.

\section{ACKNOWLEDGEMENTS}

This study was supported by the Ukrainian Science and Technology Committee under project No. 4/339.

\section{References}

Dillamore, I. L. and Roberts, W. T. (1964). Acta metall., 12, 281.

Dnieprenko, V. N., Larikov, L. N. and Stoyanova, E. N. (1982). Metallofizika, 4, 58.

Foreman, A. J. E. (1955). Acta metall., 3, 322.

Goodman, S. R. and Hu, H. (1968). Trans. Met. Soc. AIME, 242, 88.

Haeßner, F. (1963). Z. Metallk., 54, 98.

Hirsch, J. and Lücke, K. (1988). Acta metall., 36, 2883.

Karthaller, H. F. (1972). Phil. Mag., 38, 367.

Larikov, L. N. and Dnieprenko, V. N. (1979). Dopov. Akad. Nauk Ukr. SSR. Ser. A, 11, 965.

Leffers, T. (1962a). Phys. Stat. Sol., 25, 337; 1968b, Riso Rep. No. 184.

Regenet, P. J. and Stüwe, H.-P. (1963). Z. Metallk., 54, 273.

Romanov, A. E. and Vladimirov, V. I. (1983). Phys. Stat. Sol. . 78, 11. 
Takeuchi, T. (1970). J. Phys. Soc. Jap., 28, 955.

Trefilov, V. I., Mil'man, Yu. V. and Firstov, S. A. (1975). Fizicheskie osnovi prochnosti tugoplavkih materialov (Physical Basis of Strength of High-Melting-Point Metals), Naukova Dumka, Kiev. (In Russian).

Vandermeer, R. A. and McHarque, C. J. (1964). Trans. Met. Soc. AIME, 230, 667.

Wassermann, G. and Grewen, J. (1962). Texturen-Metallischer Werkatoffe. Springer-Verlag, Berlin. Wiezbanowski, K. and Jasienski, Z. (1982). Scripta metall., 16, 653.

Wierzbanowski, K., Hihi, A., Berveiller, M. and Clement, A. (1984). Proc. 7th ICOTOM, pp. 179-184. Noordwijkerhout, Netherlands Society of Materials Science.

Zolotarevski, N. Yu, Ribin, V. V. and Zhukovski, I. M. (1989). Fiz. Metallovi Metallovedenie, 67, 221. 